\title{
Parallel Transmission Electron and Atomic Force Microscopy: Direct and Repetitive Correlation of TEM and AFM images by a Novel Sample Holder
}

\author{
Alvin C. Lin* and M. Cynthia Goh* \\ * Department of Chemistry, University of Toronto, Toronto, ON, M5S 3H6
}

Transmission electron microscopy (TEM) and atomic force microscopy (AFM) are two common imaging techniques that can be used to investigate systems at the nanometre length scale. Although both techniques have been previously used in conjunction, e.g. [1], repetitive TEM and AFM have not been performed on exactly the same sample area for any system due to the different imaging conditions required by each technique. It would be useful to directly correlate TEM and AFM images, especially for more structurally complex biological samples where staining and other sample preparations may be involved. For example, in the case of a fibrillar collagen system, the relationship between the banding patterns revealed by TEM and the topographical features elucidated by AFM has remained uncertain. If both TEM and AFM images can be obtained of the exact same area of the sample, i.e. before and after staining, this relationship between images can be determined. By designing a novel sample holder that allows AFM to be performed on carbon-coated indexed TEM grids, we have been able to perform both TEM and AFM repetitively on the exact same sample area of fibrous long spacing (FLS) collagen, before and after negative-staining.

In our holder design [2], the TEM grid is positioned onto a marked centre and is secured in place by the pinning force provided by two metal clips. As shown in FIG. 1., these clips slide toward the centre and do not actually come in contact with the grid. Instead, folded strips of plastic are lowered onto the edges of the grid, holding it in place. Made of steel, the central base of the holder is secured magnetically to the AFM piezo scanner. The entire holder itself is only $\sim 2 \mathrm{~cm}$ wide and no more than $1 \mathrm{~cm}$ in height, comfortably fitting into the head of the Nanoscope III AFM setup (Digital Instruments, Santa Barbara, CA).

TEM and AFM height images of the exactly same area of FLS collagen are provided in FIG. 2. TEM images were obtained with an Hitachi H-7000 microscope (Nissei Sangyo, Rexdale, ON). Based on the correlated images, it is clear that the dark, periodic bands registered in TEM images correspond exactly to the periodic protrusions revealed in AFM height images, irrespective of negative staining. There is no need to invoke the idea of gap regions to explain the banding of dark regions observed in TEM, as previously suggested [3,4]. Thus, through correlated TEM and AFM, we have clarified the relationship between the TEM and AFM images of FLS collagen. Overall, by obtaining TEM and AFM images of the exact same sample area, the 3-D topography revealed by AFM could be used to better interpret the 2-D images provided by TEM (and vice versa) for other systems. [5]

References:

[1] Mulvaney, P. and Giersig, M., J. Chem. Soc., Faraday Trans. 92 (1996) 3137.

[2] Lin, A.C. and Goh, M.C., J. Microsc., (2002) In Press.

[3] Petruska, J.A. and Hodge, A.J., Proc. Natl. Acad. Sci. USA, 51 (1964) 871.

[4] Chapman, J.A. and Armitage, P.M., Conn. Tiss. Res., 1 (1972) 31.

[5] This research was supported by the Natural Science \& Engineering Research Council of Canada. 

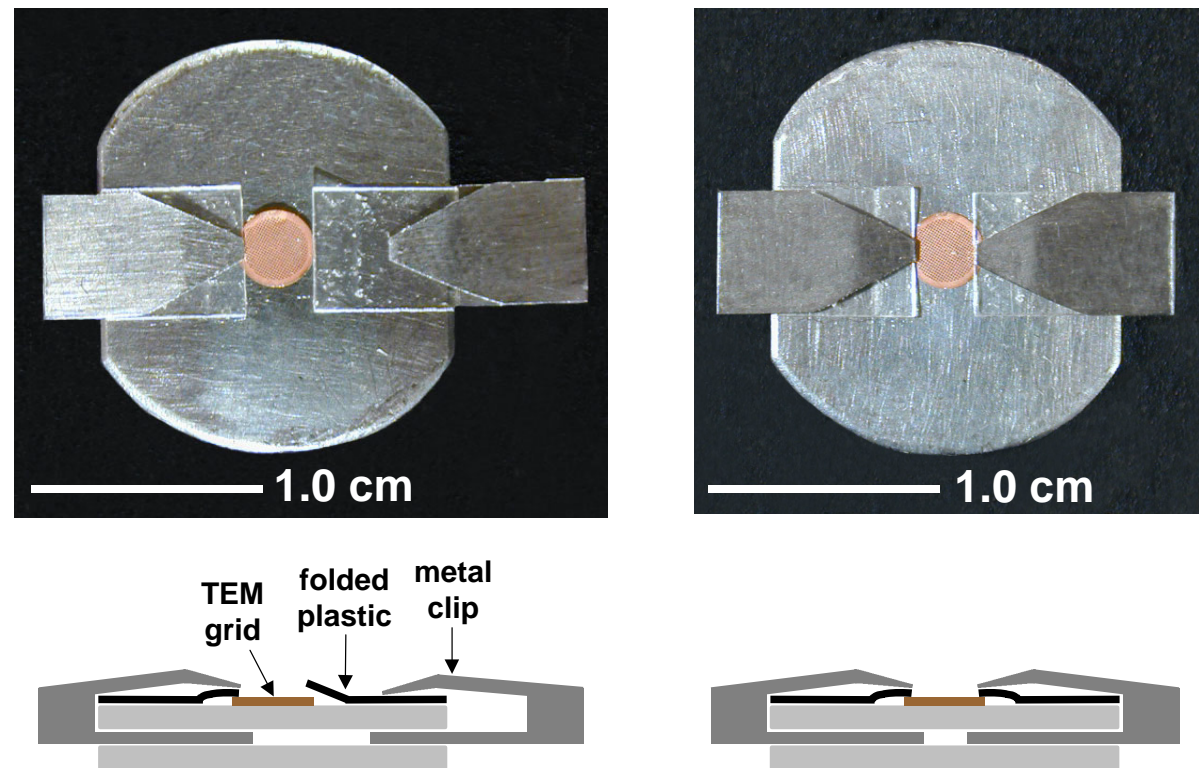

FIG. 1. Top view (above) and schematic side view (below) of the sample holder. Note that the schematic side view representations are not drawn to scale. Two metal clips slide toward the grid (left), lowering two folded plastic strips onto the edge of the grid (right).
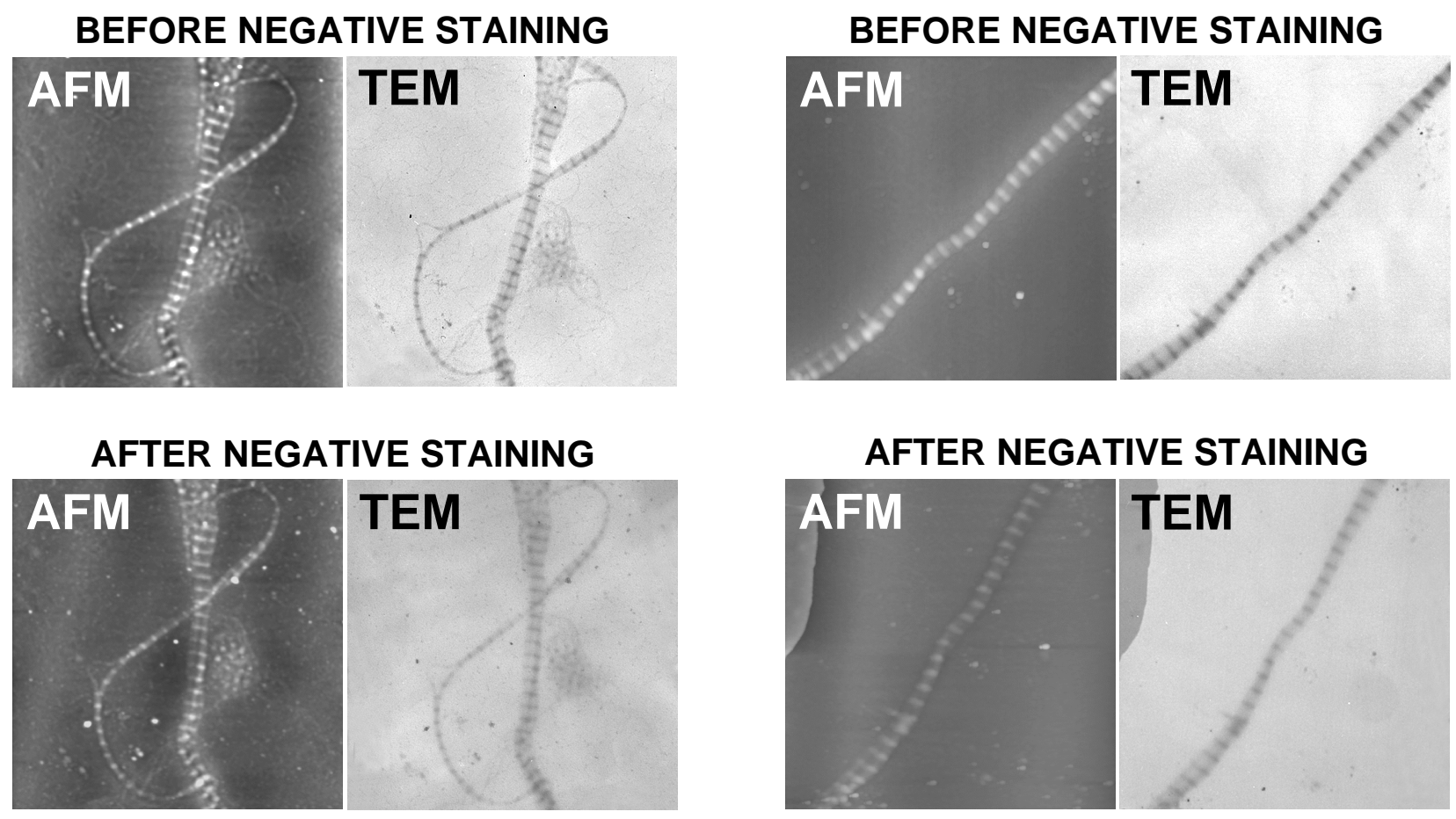

FIG. 2. Two sets of AFM height and TEM images of the exact same area of FLS collagen fibril sample taken prior to negative-staining and after negative-staining with phosphotungstic acid. Left set of images: image area $-6.0 \mu \mathrm{m}$ X $6.0 \mu \mathrm{m}$; AFM height scale: 0 (dark) to $25 \mathrm{~nm}$ (light). Right set of images: image area $-5.0 \mu \mathrm{m}$ X $5.0 \mu \mathrm{m}$; AFM height scale: 0 (dark) to $75 \mathrm{~nm}$ (light). 